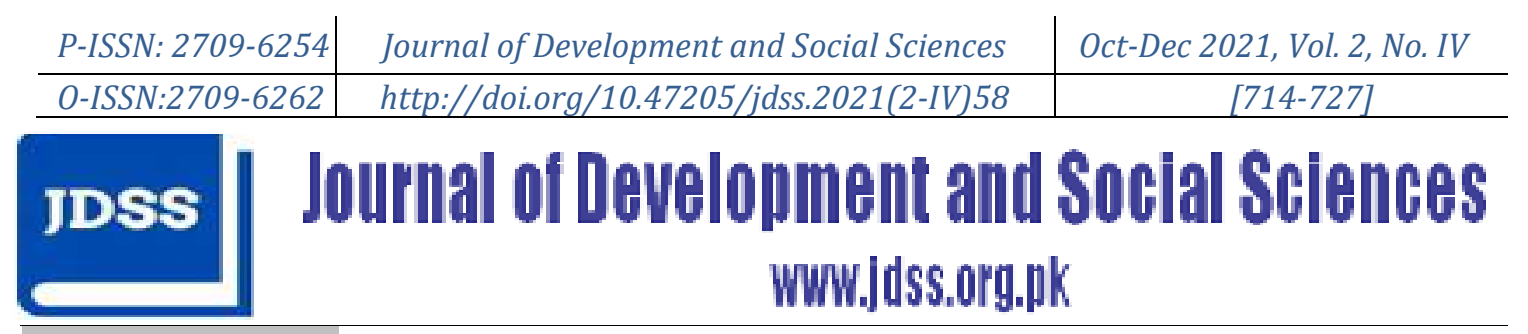

RESEARCH PAPER

\title{
Estimating Growth Model by Non-Nested Encompassing: A Cross Country Analysis
}

\author{
Benish Rashid ${ }^{1}$ Dr. Shahid Razzaque* 2 Dr. Atiq ur Rehman ${ }^{3}$
}

1. Ph. D Scholar, Pakistan Institute of Development Economics, Islamabad, Pakistan

2. Assistant Professor, Pakistan Institute of Development Economics, Islamabad, Pakistan

3. Associate Professor, Kashmir Institute of Economics, Azad Jammu and Kashmir, Pakistan

\begin{tabular}{|c|c|}
\hline API & BSTRACT \\
\hline 1 & \multirow{3}{*}{$\begin{array}{l}\text { Whether models are nested or non-nested it is important to be able } \\
\text { to compare them and evaluate their comparative results. In this } \\
\text { study six growth models have been used for analyzing the main } \\
\text { determinants of economic growth in case of cross countries, } \\
\text { therefore by using these six models we have tested them for non- } \\
\text { nested and nested encompassing through Cox test and F-test } \\
\text { respectively. Data from } 1980 \text { to } 2020 \text { were used to analyze the cross } \\
\text { country growth factors so therefore, the current study looked at } \\
\text { about forty four countries with modelling these different } \\
\text { comparative studies based on growth modelling. So, we can make } \\
\text { these six individual models and we can estimate the General } \\
\text { Unrestricted Model with the use of econometric technique of Non- } \\
\text { Nested Encompassing. By evaluating the data using the Non-Nested } \\
\text { Encompassing econometric technique, different sets of economic } \\
\text { variables has been used to evaluate which sets of the economic } \\
\text { variables are important to boost up the growth level of the country. } \\
\text { And found that in case of nested model or full model it is concluded } \\
\text { that model with lag value of GDP, trade openness, population, real } \\
\text { export, and gross fix capital formation are the main and potential } \\
\text { determinants to boost up the Economic Growth in most of the } \\
\text { countries. }\end{array}$} \\
\hline & \\
\hline $\bar{g}$ & \\
\hline
\end{tabular}

Introduction

The preceding model must explain the relevant characters, according to Hendry and Richard (1989). Pesaran claims that parametric limits prevent model derivation (1974). The "true" model's function divides the literature into two basic categories. But Hendry, Mizon, and Richard used the encompassing principle to imagine a model's success. This model should explain other theories' important traits (i.e. encompass them).The high dimensional difficulty can be met in a number of ways. This study investigates the cross-country analysis dynamics based on GDP growth potential variables.

Abou-Ali et. al. (2009). Inflation, the study determined, slows growth. Anyanwu (2014) found a link between inflation and economic growth. A study in Bangladesh 
concluded that inflation inhibits economic growth. Awan (2010) detected this relationship in Pakistan, This research tried to connect capital formation and economic growth in India. The paper explored the relationship between economic growth and capital formation. Economic growth and capital formation in Sub-Saharan Africa were explored by Uneze (2013). A bidirectional causality between private and gross capital formation was also established. Remittances have been linked to economic growth in several research (Gupta et al., 2009; Jong wanich, 2007; Stark and Lucas, 1988). The study indicated that remittances help underdeveloped countries grow. A weak finance system may allow poor countries to employ remittances to fund investment requirements. Remittances boost school enrollment and minimise child labour, according to Giuliano (2009).Human capital is statistically associated with economic growth, according to De La Fuente and Domenech (2000). Bassanini and Scarpetta (2001) found that an extra school year increased GDP by 6\%. They find that human capital adds to economic growth.

\section{Material and Methods}

\section{Nested and Non-Nested Encompassing approach}

Nestedness is defined as M1 being contained within M2, and non-nestedness is defined as the absence of any of the conditions in this definition being met by M1 and M2. When M1 and M2 do not satisfy the conditions in this definition, they are said to be nonnested.

$$
\begin{array}{ll}
M 1: & Y=X \beta+\varepsilon \ldots \ldots . . \quad M 1 \text { and } M 2 \text { (Non- Nested) } \\
\text { M2: } Y=Z \gamma+\ldots \ldots . . & \\
M^{*}: Y=X \beta+Z \gamma+W \delta+\ldots . \text { Both } M 1 \text { and } M 2 \text { (Nested) }
\end{array}
$$

\section{Encompassing And General to specific approach}

The encompassing approach connects various models. This section will use a nonnested hypothesis test to encompass. Assume we have ' $n$ ' and follow the steps below. Predict all models and note the error. The best model is the one with the least error. Basically, one model must have the least regression prediction error (Hoover et al. 1999).

$$
Y_{t}=\beta_{o}+\beta_{o} X_{1}+\beta_{1} X_{1}+\beta_{2} X_{2}+\beta_{3} X_{3}+\cdots \cdot \beta_{n} X_{n}+\mu_{t} \ldots \ldots \text { (1) }
$$

Combine the best model with other models using the hypothesis test below.

$$
\begin{aligned}
& H_{0:} \text { Model } M^{*} \text { encompasses (Model) } 1 \\
& H_{0} \text { : } \quad \text { Model } M^{*} \text { encompasses (Model) } 2 \\
& H_{0} \text { : } \quad \text { Model } M^{*} \text { encompasses (Model)' } n^{\prime}
\end{aligned}
$$

Hypothesis testing procedure is done by using below test statistics.

Cox Test

$>$ Ericson Test 


\section{Sargan Test}

\section{T-test statistics:}

$$
\boldsymbol{t} \text { - test statistic }=\frac{\overline{\boldsymbol{S}}-\boldsymbol{\mu}}{S / \sqrt{\boldsymbol{n}}}
$$

Where $\mathbf{S}$ is denoted as the slandered deviation, and this test follows the two-tail test. and t-distribution test statistics such as given:

For the linear hypothesis (F- Statistics :)

$$
F-\text { statistic }=\frac{S S E_{1}-S S E_{2} / m}{\frac{S S E_{2}}{n-K}}
$$

The restricted model has $\mathrm{k}$ parameters and $\mathrm{n}$ observations. T-tests are used to assess parameter significance, while F-tests are used to assess joint hypothesis test results. Insignificant variables will be removed from the study.

\section{Dynamic Analysis:}

Dynamic analysis tells the significant of individual estimators, The decision rule will be made using p-values provided by the F-test. For long run analysis Equation 2 is a static equation, $\quad \boldsymbol{Y}_{t}=\boldsymbol{\beta}_{o}+\boldsymbol{\beta}_{o} X_{1}+\boldsymbol{\beta}_{1} X_{1}+\boldsymbol{\beta}_{2} X_{2}+\beta_{3} X_{3}+\beta_{n} X_{n}+\mu_{t}$.

The short-term analysis shows the real picture of an economy because the effect of any variable may not be present in the current period. It therefore also includes the delay period

$$
\Delta Y_{t}=\beta_{o}+\beta_{o} \Delta X_{1}+\beta_{1} \Delta X_{1}+\beta_{2} \Delta X_{2}+\beta_{3} \Delta X_{3}+\beta_{n} \Delta X_{n}+\mu_{t \ldots(3)}
$$

Table Error! Use the Home tab to apply 0 to the text that you want to appear here.Results Sheet (Non- Nested Encompasses)

\begin{tabular}{cl}
\hline Country Name & \multicolumn{1}{c}{ Models Of Economic Growth } \\
\hline Model 1 & LnGDP =f ( FDI(inf) , T Debts, DI , Inf ) \\
\hline Model 2 & LnGDP =f ( Inf, LnTLF, TOTP, FDI (inf ), GExp) \\
\hline Model 3 & LnGDP =f ( Edu, RExp, P(remi), FDI ) \\
\hline Model 4 & LnGDP =f ( Inf, LnGCF , Rexp, P(remi) \\
\hline Model 5 & LnGDP = f ( FDI ,TOP, LG, DI , LnGCF , ) \\
\hline Model 6 & LnGDP =f ( DI, FDI, Edu, TOP) \\
\hline General Un Restricted Model (Economic Growth)
\end{tabular}

$$
\begin{aligned}
& \left.L N G D P_{t}=\beta_{o}+\beta_{1} \text { LNGDP_1 }+\beta_{2} \text { FDI (inf }\right)_{t}+\beta_{3} \text { FDI(inf) } 1_{t}+\beta_{4} \text { TOP } t+ \\
& \beta_{5} \text { TOP }_{-}{ }_{t} \beta_{6} \mathrm{LG}_{t}+\beta_{7} \mathrm{LG}_{-} 1_{t}+\beta_{8} \mathrm{DI}_{t}+\beta_{9} \mathrm{DI}_{-} 1_{t}+\beta_{10} \mathrm{LnGCF}_{t}+\beta_{11} \text { LnGCF }_{t}+ \\
& \beta_{12} \text { TDebtS }_{t}+\beta_{13} \text { TDebtS }_{-} 1_{t}+\beta_{14} \text { Inf }_{t}+\beta_{15} \text { Inf }_{-} 1_{t}+\beta_{16} \text { LnTLF }_{t}+\beta_{17} \text { LnTLF }_{-} 1_{t}+ \\
& \beta_{18} \operatorname{LnTOTP}_{t}+\beta_{19} \operatorname{LnTOTP}_{-} 1_{t}+\beta_{20} \text { Edu }_{t}+\beta_{21} \text { Edu }_{-} 1_{t}+\beta_{22} \operatorname{LnRExp}_{t}+ \\
& \beta_{23} \operatorname{LnRExp} 1_{t}+\beta_{24} \operatorname{LnGExp}{ }_{t}+\beta_{25} \operatorname{LnGExp} 1_{t}+\beta_{26} P(\text { remi })_{t}+\beta_{27} P(\text { remi })_{-} 1_{t}+ \\
& \left.\mu_{t} \ldots \ldots . . . .1\right)
\end{aligned}
$$


Table 2

Results Sheet (Sigma Values)

\begin{tabular}{|c|c|c|c|c|c|c|c|}
\hline Country Name & Model 1 & $\begin{array}{c}\text { Model } \\
2\end{array}$ & $\begin{array}{c}\text { Model } \\
3\end{array}$ & $\begin{array}{c}\text { Model } \\
4\end{array}$ & $\begin{array}{c}\text { Model } \\
5\end{array}$ & $\begin{array}{c}\text { Model } \\
6\end{array}$ & $\begin{array}{c}\text { Best } \\
\text { Model }\end{array}$ \\
\hline Argentina & 5.045 & 4.995 & 5.008 & 2.355 & 1.986 & 4.652 & Model 5 \\
\hline Australia & 0.102 & 0.076 & 0.072 & 0.073 & 0.090 & 0.091 & Model 3 \\
\hline Austria & 0.105 & 0.101 & 0.110 & 0.101 & 0.0827 & 0.096 & Model 5 \\
\hline Bangladesh & 6.646 & 1.077 & 1.027 & 0.994 & 9.813 & 9.167 & Model 4 \\
\hline Belgium & 1.723 & 1.788 & 1.993 & 1.3204 & 0.968 & 1.887 & Model 5 \\
\hline Bhutan & 0.089 & 0.056 & 0.091 & 0.082 & 0.056 & 0.081 & Model 2 \\
\hline Balgaria & 0.191 & 0.129 & 0.130 & 0.132 & 0.193 & 0.187 & Model 2 \\
\hline Brazil & 0.171 & 0.143 & 0.147 & 0.112 & 0.126 & 0.118 & Model 4 \\
\hline Canada & 0.066 & 0.073 & 0.064 & 0.045 & 0.054 & 0.065 & Model 4 \\
\hline China & 0.055 & 0.059 & 0.065 & 0.065 & 0.054 & 0.065 & Model 5 \\
\hline Chilli & 0.127 & 0.069 & 0.071 & 0.044 & 0.079 & 0.084 & Model 4 \\
\hline Denmark & 0.092 & 0.032 & 0.061 & 0.042 & 0.083 & 0.081 & Model 2 \\
\hline France & 1.256 & 1.114 & 1.314 & 1.230 & 1.244 & 1.256 & Model 2 \\
\hline Germany & 1.885 & 1.546 & 1.775 & 1.758 & 1.837 & 1.972 & Model 2 \\
\hline Ghana & 0.153 & 0.117 & 0.143 & 0.137 & 0.116 & 0.143 & Model 5 \\
\hline Hungary & 3.34837 & 2.875 & 2.988 & 2.702 & 2.882 & 2.889 & Model 4 \\
\hline India & 0.069 & 0.058 & 0.062 & 0.060 & 0.054 & 0.071 & Model 5 \\
\hline Indonesia & 0.101 & 0.132 & 0.1495 & 0.157 & 0.195 & 0.130 & Model 1 \\
\hline Iran & 0.213 & 0.260 & 0.201 & 0.254 & 0.296 & 0.194 & Model 6 \\
\hline Japan & 0.091 & 0.072 & 0.095 & 0.089 & 0.080 & 0.078 & Model 2 \\
\hline Luxembourg & 0.101 & 0.087 & 0.120 & 0.091 & 0.098 & 0.088 & Model 2 \\
\hline Malaysia & 0.070 & 0.054 & 0.089 & 0.091 & 0.073 & 0.042 & Model 6 \\
\hline Maldives & 0.089 & 0.111 & 0.093 & 0.103 & 0.120 & 0.107 & Model 1 \\
\hline Mexico & 0.121 & 0.095 & 0.137 & 0.086 & 0.087 & 0.116 & Model 4 \\
\hline Morocco & 0.441 & 0.078 & 0.096 & 0.094 & 0.264 & 0.246 & Model 6 \\
\hline Nepal & 0.067 & 0.079 & 0.070 & 0.073 & 0.075 & 0.073 & Model 1 \\
\hline Netherland & 0.098 & 0.051 & 0.063 & 0.057 & 0.099 & 0.108 & Model 2 \\
\hline Newzeland & 0.103 & 0.088 & 0.087 & 0.086 & 0.095 & 0.097 & Model 4 \\
\hline Norway & 0.083 & 0.056 & 0.057 & 0.052 & 0.069 & 0.081 & Model 4 \\
\hline Pakistan & 0.064 & 0.068 & 0.054 & 0.053 & 0.075 & 0.074 & Model 4 \\
\hline Peru & 0.109 & 0.076 & 0.094 & 0.067 & 0.076 & 0.121 & Model 4 \\
\hline Paraguay & 0.148 & 0.096 & 0.113 & 0.107 & 0.097 & 0.110 & Model 2 \\
\hline Philippines & 0.07 & 0.077 & 0.066 & 0.075 & 0.075 & 0.064 & Model 6 \\
\hline Portugal & 0.104 & 0.061 & 0.061 & 0.051 & 0.097 & 0.116 & Model 4 \\
\hline Qatar & 0.114 & 0.102 & 0.120 & 0.094 & 0.066 & 0.078 & Model 5 \\
\hline South Africa & 0.127 & 0.058 & 0.083 & 0.072 & 0.119 & 0.121 & Model 2 \\
\hline Sri Lanka & 0.041 & 0.0580 & 0.053 & 0.045 & 0.063 & 0.068 & Model 1 \\
\hline Switzerland & 0.102 & 0.054 & 0.056 & 0.048 & 0.082 & 0.098 & Model 4 \\
\hline
\end{tabular}


Estimating Growth Model by Non-Nested Encompassing: A Cross Country Analysis

\begin{tabular}{cccccccc}
\hline Sweden & 0.115 & 0.049 & 0.06 & 0.056 & 0.095 & 0.114 & Model 2 \\
\hline Turkey & 0.086 & 0.122 & 0.124 & 0.105 & 0.104 & 0.112 & Model 1 \\
\hline United States & 0.016 & 0.015 & 0.016 & 0.009 & 0.011 & 0.015 & Model 4 \\
\hline United Kingdom & 0.089 & 0.057 & 0.055 & 0.053 & 0.077 & 0.088 & Model 4 \\
\hline Uruguay & 0.099 & 0.095 & 0.128 & 0.073 & 0.076 & 0.111 & Model 4 \\
\hline
\end{tabular}

In the table above, optimal model is based on the least sigma value; this is termed variance encompassing. The model with the lowest sigma value is considered the best. Therefore, we will compare it to another models in the literature using non-nested hypothesis test

Table 3

Testing Non-Nested Hypothesis (Economic Growth)

\begin{tabular}{|c|c|c|c|c|c|c|}
\hline Argentina & $-1.524[0.1276]$ & $-1.852[0.0641$ & $-1.187[0.2352]$ & $-1.274[0.2026]$ & .. & $-2.535[0.0112]^{*}$ \\
\hline Australia & $-1.084[0.2784]$ & $-2.702[0.0069]^{* *}$ & .. & $-3.029[0.0025]^{* *}$ & $-6.215[0.0000]^{* *}$ & $-4.222[0.0000]^{* *}$ \\
\hline Austria & $1.056[0.3842]$ & $0.1508[0.8802]$ & $0.5088[0.6109]$ & $1.587[0.1124]$ &.. & $0.6202[0.5351]$ \\
\hline Bangladesh & $-2.902[0.0037]^{* *}$ & $-3.077[0.0021]^{* *}$ & $-5.175[0.0000]^{* *}$ & .. & $-15.46[0.0000]^{* *}$ & $-11.88[0.0000]^{* *}$ \\
\hline Belgium & $0.4812[0.6303]$ & $1.607[0.1081]$ & $1.958[0.0503]$ & $-0.1123[0.9106]$ &.. & $-0.1571[0.8752]$ \\
\hline Bhutan & $0.6420[0.5209]$ &.. & $-0.4124[0.6801]$ & $-0.2139[0.8306]$ & $-6.122[0.0000]^{* *}$ & $-4.037[0.0001]^{* *}$ \\
\hline Balgaria & $-2.026[0.0427]^{*}$ &.. & $-2.198[0.0279]^{*}$ & $-2.070[0.0385]^{*}$ & $-1.239[0.2153]$ & $-1.816[0.0694]$ \\
\hline Brazil & $-3.095[0.0020]^{* *}$ & $-1.190[0.2341]$ & $-3.083[0.0021]^{* *}$ & ... & $-2.948[0.0032]^{* *}$ & $-5.849[0.0000]^{* *}$ \\
\hline Canada & $1.049[0.2942]$ & $1.602[0.1091]$ & $0.002086[0.9983]$ & ... & $-2.072[0.0383]^{*}$ & $0.09950[0.9207]$ \\
\hline China & $-0.8182[0.4132]$ & $-1.953[0.0508]$ & $-0.3222[0.7473]$ & $-0.4732[0.6361]$ & .. & $0.01299[0.9896$ \\
\hline Chilli & $2.641[0.0083]^{* *}$ & $8.537[0.0000]^{* *}$ & $-4.078[0.0000]^{* *}$ & ... & $0.3567[0.7213]$ & $-2.246[0.0247]^{*}$ \\
\hline Denmark & $-5.006[0.0000]^{* *}$ & ... & $-0.2485[0.8038]$ & $-3.033[0.0024]^{* *}$ & $-0.5273[0.5980]$ & $0.4855[0.6273]$ \\
\hline France & $-3.531[0.0000]^{* *}$ & ... & $0.1443[0.8853]$ & $-4.513[0.0000]^{* *}$ & $-3.443[0.0006]^{* *}$ & $-1.980[0.0477]^{*}$ \\
\hline Germany & $-1.321[0.0009]^{* *}$ & .. & $-4.731[0.0000]^{* *}$ & $-5.836[0.0000]^{* *}$ & $-2.639[0.0083]^{* *}$ & $-0.7262[0.4677]$ \\
\hline Ghana & $-1.263[0.2066]$ & $-5.805[0.0000]^{* *}$ & $-0.6658[0.5055]$ & $-1.160[0.2461]$ &.. & $-0.02662[0.9788]$ \\
\hline Hungary & $-1.733[0.0832]$ & $-6.007[0.0000]^{* *}$ & $-3.311[0.0009]^{* *}$ & ... & $-4.349[0.0000]^{* *}$ & $-2.338[0.0194]^{*}$ \\
\hline India & $0.9661[0.3340]$ & $-3.861[0.0001]^{* *}$ & $-0.1389[0.8895]$ & $-0.7905[0.4293]$ & .. & $0.5447[0.5860]$ \\
\hline Indonesia & .. & $-1.004[0.3151]$ & $-0.7010[0.4833]$ & $-4.440[0.0000]^{* *}$ & $-2.245[0.0248]^{*}$ & $-4.989[0.0000]^{* *}$ \\
\hline Iran & $-7.054[0.0000]^{* *}$ & $-11.88[0.0000]^{* *}$ & $-8.914[0.0000]^{* *}$ & $-8.080[0.0000]^{* *}$ & $-8.456[0.0000]^{* *}$ &.. \\
\hline Japan & $0.1379[0.8903]$ & ... & $-0.8707[0.3839]$ & $-1.175[0.2401]$ & $-1.313[0.1890]$ & $-1.810[0.0702]$ \\
\hline Luxembourg & $4.350[0.0000]^{* *}$ & .. & $0.5534[0.5800]$ & $-2.618[0.0088]^{* *}$ & $-0.8845[0.3764]$ & $-6.001[0.0000]^{* *}$ \\
\hline Malaysia & $3.420[0.0006]^{* *}$ & $-9.715[0.0000]^{* *}$ & $-1.041[0.2980]$ & $-3.136[0.0017]^{* *}$ & $-4.076[0.0000]^{* *}$ &.. \\
\hline Maldives & .. & $-2.826[0.0047]^{* *}$ & $-9.967[0.0000]^{* *}$ & $-4.829[0.0000]^{* *}$ & $0.1378[0.8904]$ & $-8.152[0.0000]^{* *}$ \\
\hline Mexico & $-0.4716[0.6372]$ & $-2.028[0.0426]^{*}$ & $1.267[0.2053]$ & ... & $-3.259[0.0011]^{* *}$ & $1.281[0.2000]$ \\
\hline Morocco & $-4.558[0.0000]^{* *}$ & $-22.30[0.0000]^{* *}$ & $-17.06[0.0000]^{* *}$ & $-18.35[0.0000]^{* *}$ & $-1.181[0.2376]$ & .. \\
\hline Nepal & .. & $-0.3532[0.7239]$ & $-4.032[0.0001]^{* *}$ & $-3.392[0.0007]^{* *}$ & $-1.753[0.0795]$ & $-2.498[0.0125]^{*}$ \\
\hline Netherland & $0.6651[0.5060]$ & ... & $-0.6500[0.5157]$ & $-2.638[0.0083]^{* *}$ & $-0.8125[0.4165]$ & $-0.4269[0.6695]$ \\
\hline Newzeland & $1.706[0.0879]$ & $-2.000[0.0455]^{*}$ & $-2.093[0.0363]^{*}$ & .. & $-7.903[0.0000]^{* *}$ & $-4.379[0.0000]^{* *}$ \\
\hline Norway & $21.015[0.0071]^{* *}$ & $-1.270[0.2040]$ & $-1.975[0.0483]^{*}$ & .. & $-2.008[0.0447]^{*}$ & $0.1659[0.8682]$ \\
\hline Pakistan & $-3.263[0.0011]^{* *}$ & $-0.03716[0.9704]$ & $-2.590[0.0096]^{* *}$ & ... & $1.351[0.1766]$ & $-0.1120[0.9109]$ \\
\hline Peru & $-0.7935[0.4275]$ & $-4.416[0.0000]^{* *}$ & $-2.149[0.0316]^{*}$ & & $-5.209[0.0000]^{* *}$ & $-0.8842[0.3766]$ \\
\hline Paraguay & $-0.4010[0.6885]$ & & $-2.149[0.0316]^{*}$ & $-3.405[0.0007]^{* *}$ & $-6.861[0.0000]^{* *}$ & $-3.400[0.0007]^{* *}$ \\
\hline Philippines & $-1.829[0.0674]$ & $-0.5689[0.5694]$ & $-2.026[0.0428]^{*}$ & $-3.048[0.0023]^{* *}$ & $-2.373[0.0176]^{*}$ & .. \\
\hline Portugal & $1.790[0.0735]$ & $1.790[0.0735]$ & $4.2524[0.0244]^{*}$ & .. & $-1.821[0.0686]$ & $0.9842[0.3250]$ \\
\hline Qatar & $3.4821[0.0142]^{*}$ & $-3.655[0.0003]^{* *}$ & $-2.405[0.0162]^{*}$ & $-3.467[0.0005]^{* *}$ &.. & $-0.6991[0.4845]$ \\
\hline South Africa & $0.9443[0.3450]$ & .. & $-0.6909[0.4896]$ & $-2.839[0.0045]^{* *}$ & $-2.271[0.0231]^{*}$ & $-2.041[0.0412]^{*}$ \\
\hline Sri Lanka & & $-3.997[0.0001]^{* *}$ & $-4.961[0.0000]^{* *}$ & $-4.961[0.0000]^{* *}$ & $-0.2751[0.7832]$ & $0.5417[0.5880]$ \\
\hline Switzerland & $0.8319[0.4055]$ & $-3.749[0.0002]^{* *}$ & $-1.117[0.2638]$ & .. & $-0.4449[0.6564]$ & $0.2620[0.7933]$ \\
\hline
\end{tabular}




\begin{tabular}{lllllll}
\hline Sweden & $3.430[0.0006]^{* *}$ &.. & $-0.06186[0.9507]$ & $-4.363[0.0000]^{* *}$ & $-0.3404[0.7335]$ & $4.1073[0.0273]^{*}$ \\
\hline Turkey &.. & $-1.420[0.1556]$ & $-7.584[0.0000]^{* *}$ & $-3.263[0.0011]^{* *}$ & $-2.807[0.0050]^{* *}$ & $-5.444[0.0000]^{* *}$ \\
\hline United States & $0.1010[0.9195]$ & $0.1902[0.8492]$ & $0.5792[0.5625]$ &.. & $-0.02044[0.9837]$ & $1.525[0.1273]$ \\
\hline United Kingdi & $5.807[0.0000]^{* *}$ & $-2.034[0.0419]^{*}$ & $-7.078[0.0000]^{* *}$ &.. & $-1.310[0.1901]$ & $0.7930[0.4278]$ \\
\hline Uruguay & $-5.262[0.0000]^{* *}$ & $-2.402[0.0163]^{*}$ & $-0.5995[0.5489]$ & & $-11.04[0.0000]^{* *}$ & $-2.714[0.0066]^{* *}$ \\
\hline
\end{tabular}

Economic growth is estimated by using the Non-Nested Encompassing Model. The current study covered cross country analysis of 44 nations from 1980 to 2020. In the above table of non-nested hypothesis testing test statistics which can reject or in the favor of acceptance of null hypothesis on the based of cox test statistics will be consider as a bench mark. So we will make union of rejected model with model along with best model selected from the least sigma value. After estimation of GUM model we will apply general to specific approach to get parsimonious model for GDP.

Table 4

Final Model from Non-Nested Encompassing (Economic Growth)

\begin{tabular}{|c|c|c|c|c|c|c|c|c|}
\hline $\begin{array}{l}\text { Country } \\
\text { Name }\end{array}$ & Constant & LNGDP_1 & FDI(inf) & FDI(inf)_1 & TOP & TOP_1 & LG & LG_1 \\
\hline Argentina & $\begin{array}{c}-4.692 \\
(0.012) \\
\end{array}$ & $\begin{array}{c}0.927 \\
(0.0000) \\
\end{array}$ & .. & $\begin{array}{c}0.021 \\
(0.0411) \\
\end{array}$ & $-9.127 \quad(0.0000)$ & $\begin{array}{c}9.557 \\
(0.0000) \\
\end{array}$ & .. & $\begin{array}{r}-23.419 \\
(0.0001) \\
\end{array}$ \\
\hline Australia & $\begin{array}{c}-15.647 \\
(0.0010) \\
\end{array}$ & $\begin{array}{c}0.614 \\
(0.0000) \\
\end{array}$ & & .. & 1.918 (0.0389) & $\begin{array}{c}2.447 \\
(0.0011) \\
\end{array}$ & .. & .. \\
\hline Austria & .. & $\begin{array}{c}0.437 \\
0.0009 \\
\end{array}$ & .. &. & $-3.650 \quad 0.0001$ & .. & .. & .. \\
\hline $\begin{array}{c}\text { Banglades } \\
\mathbf{h}\end{array}$ & $\begin{array}{c}38.729 \\
(0.0000) \\
\end{array}$ & $\begin{array}{c}0.135 \\
(0.0355) \\
\end{array}$ & .. & $\begin{array}{c}-0.041 \\
(0.0085) \\
\end{array}$ & $-9.949 \quad(0.0000)$ & .. & $\begin{array}{c}0.729 \\
(0.0006) \\
\end{array}$ & .. \\
\hline Belgium & .. & $\begin{array}{c}0.909 \\
(0.0000)\end{array}$ & .. &.. & .. & .. & .. & .. \\
\hline Bhutan & .. & $\begin{array}{c}0.960 \\
(0.0000) \\
\end{array}$ & $\begin{array}{c}0.0152 \\
(0.0005) \\
\end{array}$ & .. & $-0.107 \quad(0.0071)$ & .. & .. & .. \\
\hline Balgeria &.. & $\begin{array}{c}0.245 \\
(0.0124) \\
\end{array}$ &. &. &. & .. &. & .. \\
\hline Brazil &. & $\begin{array}{c}0.933 \\
(0.0000) \\
\end{array}$ & .. &.. & $\begin{array}{c}-14.964 \\
(0.0000) \\
\end{array}$ & $\begin{array}{l}13.371 \\
0.0000 \\
\end{array}$ & .. & .. \\
\hline Canada & $\begin{array}{c}5.731 \\
(0.0085) \\
\end{array}$ & $\begin{array}{c}0.821 \\
(0.0000) \\
\end{array}$ & .. &. & $\begin{array}{c}-13.495 \\
(0.0000) \\
\end{array}$ & $\begin{array}{c}10.163 \\
(0.0000) \\
\end{array}$ & .. & .. \\
\hline China & $\begin{array}{c}-1.645 \\
(0.0017) \\
\end{array}$ & $\begin{array}{c}0.871 \\
(0.0000) \\
\end{array}$ &. & $\begin{array}{c}0.040 \\
(0.0000) \\
\end{array}$ & .. & .. & .. & .. \\
\hline Chilli & $\begin{array}{c}-41.445 \\
(0.0048) \\
\end{array}$ & $\begin{array}{c}0.646 \\
(0.0000) \\
\end{array}$ &.$\cdot$ & .. & .. & .. & .. &. \\
\hline Denmark & $\begin{array}{c}9.5 .38 \\
(0.0000)\end{array}$ & $\begin{array}{c}0.434 \\
(0.0000) \\
\end{array}$ &.. &.. & .. & .. & .. &.. \\
\hline France & .. & $\begin{array}{c}0.528 \\
(0.0006)\end{array}$ & $\begin{array}{c}-0.002 \\
(0.0232)\end{array}$ & .. & $\begin{array}{c}-17.715 \\
(0.0000)\end{array}$ & $\begin{array}{c}9.553 \\
(0.0004) \\
\end{array}$ & $\begin{array}{c}-0.262 \\
(0.0215)\end{array}$ & .. \\
\hline Germany & $\begin{array}{r}28.1776 \\
(0.0164) \\
\end{array}$ & $\begin{array}{c}0.041 \\
(0.0123) \\
\end{array}$ & $\begin{array}{c}0.006 \\
(0.0000) \\
\end{array}$ & $\begin{array}{c}0.004 \\
90.00050 \\
\end{array}$ & $\begin{array}{c}-13.087 \\
(0.0000) \\
\end{array}$ & C & C & $\begin{array}{c}-3.551 \\
(0.0000) \\
\end{array}$ \\
\hline Ghana & .. & $\begin{array}{c}0.601 \\
(0.0000) \\
\end{array}$ & .. &. & $\begin{array}{c}-8.006 \\
(0.000) 0 \\
\end{array}$ & $\begin{array}{c}4.710 \\
0.0029 \\
\end{array}$ &.. &. \\
\hline Hungary & $\begin{array}{c}20.004 \\
(0.000) 0 \\
\end{array}$ & $\begin{array}{c}0.132 \\
(0.0003) \\
\end{array}$ & & & $\begin{array}{c}-11.704 \\
(0.0000) \\
\end{array}$ & $\begin{array}{c}0.733 \\
0.0305\end{array}$ & & \\
\hline India & $\begin{array}{c}30.276 \\
(0.0003) \\
\end{array}$ & $\begin{array}{c}0.3034 \\
(0.0008) \\
\end{array}$ &. & .. &.. &.. &. &.. \\
\hline Indonesia & 18.454 & 0.221 & 0.027 &.. & -10.919 & ... & .. & -1.458 \\
\hline
\end{tabular}


Estimating Growth Model by Non-Nested Encompassing: A Cross Country Analysis

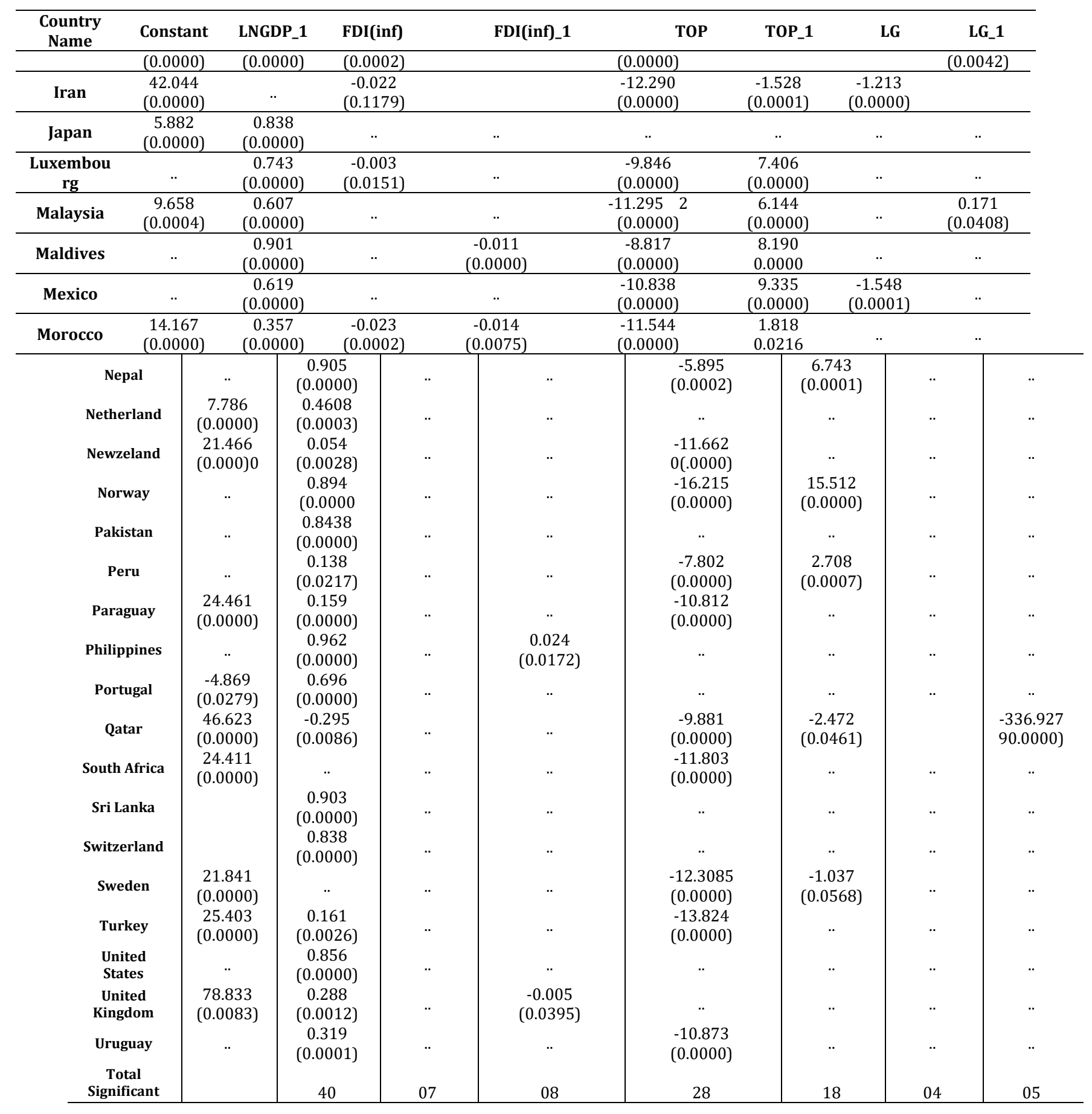




\begin{tabular}{|c|c|c|c|c|c|c|c|c|c|c|}
\hline $\begin{array}{l}\text { Country } \\
\text { Name }\end{array}$ & DI & DI_1 & LnGCF & LnGCF_1 & TDebtS & $\begin{array}{l}\text { TDebtS } \\
1\end{array}$ & Inf & Inf_1 & LnTLF & LnTLF_1 \\
\hline Argentina &.. &.. & $\begin{array}{l}1.090 \\
(0.0000)\end{array}$ & $\begin{array}{l}-0.847 \\
(0.0000)\end{array}$ & .. & .. & .. &.. &.. & .. \\
\hline Australia & $\begin{array}{l}0.034 \\
(0.0000)\end{array}$ &.. & .. & .. & .. & .. & $\begin{array}{l}-0.035 \\
(0.0001)\end{array}$ & $\begin{array}{l}-0.018 \\
(0.0104)\end{array}$ &.. & \\
\hline Austria & $\begin{array}{l}0.039 \\
0.0324\end{array}$ & .. & $\begin{array}{l}0.882 \\
0.0000\end{array}$ & .. & .. & .. & .. & .. & .. & .. \\
\hline Bangladesh & .. & .. & $\begin{array}{l}0.761 \\
(0.0000)\end{array}$ & $\begin{array}{l}-0.288 \\
(0.0412)\end{array}$ & .. & .. & $\begin{array}{l}0.004 \\
(0.0061)\end{array}$ & $\begin{array}{l}0.045 \\
(0.0065)\end{array}$ & .. & .. \\
\hline Belgium & .. & .. & $\begin{array}{l}0.756 \\
(0.0016)\end{array}$ & $\begin{array}{l}-0.661 \\
(0.0043)\end{array}$ & .. & .. &.. &.. & .. & .. \\
\hline Bhutan & .. & $\begin{array}{l}0.019 \\
(0.0019)\end{array}$ & $\begin{array}{l}0.051 \\
(0.0001)\end{array}$ &. & .. & .. & .. & $\begin{array}{l}-0.008 \\
(0.0004)\end{array}$ & .. & .. \\
\hline Balgeria & .. & .. & .. & .. & $\begin{array}{l}-0.014 \\
(0.0480)\end{array}$ & $\begin{array}{l}0.013 \\
(0.0274)\end{array}$ & .. & .. & $\begin{array}{l}-0.110 \\
(0.0004)\end{array}$ & .. \\
\hline Brazil & $\begin{array}{l}2.114 \\
(0.0000)\end{array}$ & .. & $\begin{array}{l}0.703 \\
(0.0000)\end{array}$ & $\begin{array}{l}-0.599 \\
(0.0000)\end{array}$ & .. &. & .. & .. & .. & .. \\
\hline Canada & .. & .. & $\begin{array}{l}0.301 \\
(0.0000)\end{array}$ & $\begin{array}{l}-0.276 \\
(0.0000)\end{array}$ & .. & .. & .. & .. & .. & .. \\
\hline China & $\begin{array}{l}0.011 \\
(0.0164)\end{array}$ & .. &.. & .. & .. & .. & .. & .. & .. & .. \\
\hline Chilli & .. & $\begin{array}{c}-0.002 \\
0.0085\end{array}$ & $\begin{array}{l}0.305 \\
(0.0000)\end{array}$ & .. & $\begin{array}{l}8.103 \\
(0.0003)\end{array}$ & $\begin{array}{l}-8.290 \\
(0.0002)\end{array}$ & .. & $\begin{array}{l}0.011 \\
(0.0000)\end{array}$ & .. & .. \\
\hline Denmark & $\begin{array}{l}-0.788 \\
(0.0169)\end{array}$ & $\begin{array}{l}0.902 \\
(0.0032)\end{array}$ & $\begin{array}{l}-0.179 \\
(0.0029)\end{array}$ & $\begin{array}{l}0.201 \\
(0.0005)\end{array}$ & $\begin{array}{l}-4.962 \\
(0.0000)\end{array}$ & $\begin{array}{l}4.962 \\
(0.0000)\end{array}$ & $\begin{array}{l}-0.035 \\
0.0000\end{array}$ & .. & $\begin{array}{l}0.080 \\
(0.0001)\end{array}$ & .. \\
\hline France & .. & $\begin{array}{l}0.011 \\
(0.0422)\end{array}$ & $\begin{array}{l}0.332 \\
(0.0006)\end{array}$ & $\begin{array}{l}-0.329 \\
(0.0002)\end{array}$ & $\begin{array}{l}72.803 \\
(0.0020)\end{array}$ & $\begin{array}{l}-72.805 \\
(0.0020)\end{array}$ & $\begin{array}{l}0.013 \\
(0.0000)\end{array}$ & $\begin{array}{l}-0.010 \\
(0.0033)\end{array}$ & .. & .. \\
\hline Germany & .. & $\begin{array}{l}-1.368 \\
(0.0064)\end{array}$ & $\begin{array}{l}-0.160 \\
(0.0009)\end{array}$ & $\begin{array}{l}0.084 \\
(0.0119)\end{array}$ & $\begin{array}{l}-1.345 \\
(0.0051)\end{array}$ & .. & .. &. & $\begin{array}{l}0.394 \\
(0.0000)\end{array}$ & .. \\
\hline Ghana &.. &.. & $\begin{array}{l}-0.077 \\
(0.0000)\end{array}$ & .. & .. & .. & .. &.. &.. & .. \\
\hline Hungary & & & $\begin{array}{l}0.231 \\
(0.0000)\end{array}$ & $\begin{array}{l}-0.110 \\
(0.0163)\end{array}$ & .. & .. & $\begin{array}{l}-0.001 \\
(0.0435)\end{array}$ & .. & .. & .. \\
\hline India &.. &.. & $\begin{array}{l}0.699 \\
(0.000) 0\end{array}$ &.. & .. & .. & .. & .. & $\begin{array}{l}-0.032 \\
(0.00030\end{array}$ & .. \\
\hline Indonesia & $\begin{array}{l}-0.007 \\
(0.0007)\end{array}$ & $\begin{array}{l}0.004 \\
(0.0277)\end{array}$ & $\begin{array}{l}-0.004 \\
0.7355\end{array}$ & $\begin{array}{l}0.059 \\
(0.0015)\end{array}$ & .. & .. & .. & .. &. & .. \\
\hline Iran & $\begin{array}{l}-0.009 \\
(0.0438)\end{array}$ & .. & .. & $\begin{array}{l}0.010 \\
(0.0055)\end{array}$ & $\begin{array}{l}-0.025 \\
(0.0000)\end{array}$ & .. & .. & .. & $\begin{array}{l}-0.150 \\
(0.0000)\end{array}$ & $\begin{array}{l}-0.102 \\
(0.0000)\end{array}$ \\
\hline Japan & .. & .. & .. &. & .. & .. & .. & $\begin{array}{l}-0.031 \\
(0.0107)\end{array}$ & .. & .. \\
\hline Luxembourg & .. & .. & .. & $\begin{array}{c}0.076 \\
(0.0804)\end{array}$ & .. & .. & $\begin{array}{l}-0.004 \\
(0.0912)\end{array}$ &.. & .. &.. \\
\hline Malaysia & .. & .. & $\begin{array}{l}0.196 \\
(0.0000)\end{array}$ & $\begin{array}{l}-0.137 \\
(0.0002)\end{array}$ & .. & .. & .. & .. & .. & .. \\
\hline Maldives & .. & .. &.. &.. & .. & .. & .. & .. & .. & .. \\
\hline Mexico & .. & .. & $\begin{array}{l}0.367 \\
(0.0007)\end{array}$ & .. & .. & .. & $\begin{array}{l}-0.004 \\
(0.0000)\end{array}$ & $\begin{array}{l}0.003 \\
(0.0001)\end{array}$ & .. & .. \\
\hline Morocco & .. & .. & $\begin{array}{l}0.388 \\
(0.0000)\end{array}$ & $\begin{array}{l}-0.291 \\
(0.0024)\end{array}$ & $\begin{array}{l}0.011 \\
(0.0290)\end{array}$ & .. & .. & .. & $\begin{array}{l}-0.035 \\
(0.0150)\end{array}$ & .. \\
\hline Nepal & .. & .. & .. & .. & .. & .. & .. & .. &.. & .. \\
\hline Netherland & .. & .. & .. & .. & .. & .. & $\begin{array}{l}-0.027 \\
(0.0003)\end{array}$ & .. & $\begin{array}{l}-0.171 \\
(0.0002)\end{array}$ & .. \\
\hline Newzeland & .. & .. & .. & .. & .. & .. & .. & .. & .. & .. \\
\hline Norway & .. & .. & $\begin{array}{l}0.518 \\
(0.0000)\end{array}$ & $\begin{array}{l}-0.407 \\
(0.0000)\end{array}$ & .. & .. & .. & .. & .. & .. \\
\hline Pakistan & .. & .. & 0.312 & -0.331 & .. & .. & .. & .. & .. & .. \\
\hline
\end{tabular}




\begin{tabular}{|c|c|c|c|c|c|c|c|c|c|c|}
\hline $\begin{array}{l}\text { Country } \\
\text { Name }\end{array}$ & DI & DI_1 & LnGCF & LnGCF_1 & TDebtS & $\begin{array}{l}\text { TDebtS } \\
1\end{array}$ & Inf & Inf_1 & LnTLF & LnTLF_1 \\
\hline & & & $(0.0308)$ & $(0.0228)$ & & & & & & \\
\hline Peru & .. & .. & $\begin{array}{l}0.414 \\
(0.0000)\end{array}$ & .. & .. & .. & $\begin{array}{l}4.173 \\
(0.0000)\end{array}$ & .. & .. & .. \\
\hline Paraguay & $\begin{array}{l}-0.0075 \\
(0.0000)\end{array}$ & .. & $\begin{array}{l}0.255 \\
(0.0000)\end{array}$ & .. & .. & .. & $\begin{array}{l}0.003 \\
(0.0031)\end{array}$ & .. & .. & .. \\
\hline Philippines & .. & .. & $\begin{array}{l}0.260 \\
(0.0000)\end{array}$ & $\begin{array}{l}-0.260 \\
(0.0000)\end{array}$ & .. & .. & .. & .. & .. & .. \\
\hline Portugal & .. & .. & $\begin{array}{l}0.2578 \\
(0.0003)\end{array}$ & .. & .. & .. & $\begin{array}{l}-0.016 \\
(0.0002)\end{array}$ & $\begin{array}{l}0.015 \\
(0.0017)\end{array}$ & .. & .. \\
\hline Qatar & & & $\begin{array}{l}-0.163 \\
(0.00340\end{array}$ & $\begin{array}{l}0.167 \\
(0.0036)\end{array}$ & .. & .. &. &. & $\begin{array}{l}26.890 \\
(0.0000)\end{array}$ & $\begin{array}{c}-26.8992 \\
(0.0000)\end{array}$ \\
\hline South Africa & .. & .. & $\begin{array}{l}0.255 \\
90.0000)\end{array}$ & .. & .. & .. & $\begin{array}{l}-0.008 \\
(0.0001)\end{array}$ & .. & $\begin{array}{l}0.090 \\
(0.0000)\end{array}$ & .. \\
\hline Sri Lanka & $\begin{array}{l}-0.004 \\
0.0189\end{array}$ & $\begin{array}{l}0.010 \\
0.0000\end{array}$ & .. & .. & $\begin{array}{l}-0.011 \\
(0.0083)\end{array}$ & $\begin{array}{l}-0.013 \\
(0.0027)\end{array}$ & $\begin{array}{l}0.004 \\
(0.0010)\end{array}$ & $\begin{array}{l}-0.004 \\
(0.0004)\end{array}$ & .. & $\begin{array}{l}-0.050 \\
(0.0716)\end{array}$ \\
\hline Switzerland & .. & .. & $\begin{array}{l}0.643 \\
(0.0006)\end{array}$ & $\begin{array}{l}-0.530 \\
(0.0106)\end{array}$ & .. & .. &. &. & .. & .. \\
\hline Sweden & .. & .. & .. & $\begin{array}{l}-0.064 \\
(0.0492)\end{array}$ & .. & .. & .. & $\begin{array}{l}0.003 \\
(0.0013)\end{array}$ & .. & $\begin{array}{l}0.510 \\
(0.0000)\end{array}$ \\
\hline Turkey & $\begin{array}{l}-0.002 \\
(0.0041)\end{array}$ & $\begin{array}{l}0.002 \\
(0.0007)\end{array}$ & $\begin{array}{l}0.086 \\
(0.0117)\end{array}$ & $\begin{array}{l}-0.108 \\
(0.0026)\end{array}$ & $\begin{array}{l}-0.020 \\
(0.0000)\end{array}$ & .. & .. &.. & .. &.. \\
\hline United States & .. & .. & $\begin{array}{l}0.206 \\
0.0000\end{array}$ & $\begin{array}{l}-0.099 \\
(0.0017)\end{array}$ & .. & .. & .. & .. & .. & .. \\
\hline $\begin{array}{l}\text { United } \\
\text { Kingdom }\end{array}$ & .. & $\begin{array}{l}0.018 \\
(0.0004)\end{array}$ & $\begin{array}{l}0.601 \\
(0.0000)\end{array}$ & .. & $\begin{array}{l}0.018 \\
(0.0589)\end{array}$ & .. & .. & $\begin{array}{l}0.012 \\
(0.0545)\end{array}$ & .. & .. \\
\hline Uruguay & $\begin{array}{l}-0.037 \\
(0.0001)\end{array}$ & $\begin{array}{l}-0.034 \\
(0.0000)\end{array}$ & $\begin{array}{l}0.246 \\
(0.0017)\end{array}$ & $\begin{array}{l}-0.301 \\
(0.0001)\end{array}$ & $\begin{array}{l}71.638 \\
(0.0000)\end{array}$ & $\begin{array}{l}-72.200 \\
(0.0000)\end{array}$ & $\begin{array}{l}0.0053 \\
(0.0002)\end{array}$ & .. & $\begin{array}{l}-0.031 \\
(0.0012)\end{array}$ & .. \\
\hline $\begin{array}{l}\text { Total } \\
\text { Significant }\end{array}$ & 11 & 10 & 31 & 19 & 11 & 09 & 14 & 11 & 10 & 04 \\
\hline
\end{tabular}

\begin{tabular}{|c|c|c|c|c|c|c|c|c|c|c|}
\hline $\begin{array}{l}\text { Country } \\
\text { Name }\end{array}$ & LnTOTP & LnTOTP_1 & Edu & Edu_1 & LnRExp & $\begin{array}{l}\text { LnRExp } \\
\_1\end{array}$ & GEXP & GEXP_1 & P(remi) & P(remi)_1 \\
\hline Argentina &.. & .. & $\begin{array}{l}-0.047 \\
(0.0118)\end{array}$ &.. & .. & .. & $\begin{array}{l}-0.022 \\
(0.0064)\end{array}$ &.. &.. &.. \\
\hline Australia & $\begin{array}{l}0.773 \\
(0.0171)\end{array}$ & .. & $\begin{array}{l}-0.135 \\
(0.0004)\end{array}$ & .. & $\begin{array}{l}0.538 \\
(0.0000)\end{array}$ & $\begin{array}{l}-0.312 \\
(0.0026)\end{array}$ & .. &.. & .. & .. \\
\hline Austria &.. & .. & .. & .. & .. &.. & .. & .. & .. & .. \\
\hline Bangladesh & $\begin{array}{l}-13.474 \\
(0.0069)\end{array}$ & $\begin{array}{l}12.117 \\
(0.0129)\end{array}$ & .. & .. & $\begin{array}{l}0.683 \\
(0.0000)\end{array}$ & .. & .. & $\begin{array}{l}-0.002 \\
(0.0047)\end{array}$ & $\begin{array}{l}0.0089 \\
(0.0636)\end{array}$ & .. \\
\hline Belgium & .. & .. & .. & .. & .. & .. & .. & .. & .. & .. \\
\hline Bhutan & .. & .. & .. & .. &.. & .. & $\begin{array}{l}0.002 \\
(0.0051)\end{array}$ &.. & .. &.. \\
\hline Balgeria & .. & $\begin{array}{l}0.318 \\
(0.0000)\end{array}$ & $\begin{array}{l}-0.037 \\
(0.0153)\end{array}$ & .. & $\begin{array}{l}0.645 \\
(0.0000)\end{array}$ & .. & $\begin{array}{l}0.004 \\
(0.0100)\end{array}$ & .. & .. & .. \\
\hline Brazil & .. & .. & $\begin{array}{l}-0.041 \\
(0.0002)\end{array}$ & $\begin{array}{l}0.034 \\
(0.0015)\end{array}$ & $\begin{array}{l}0.927 \\
(0.0000)\end{array}$ & $\begin{array}{l}-0.848 \\
(0.0000)\end{array}$ & .. & .. & .. & $\begin{array}{l}0.023 \\
(0.0051)\end{array}$ \\
\hline Canada & .. & .. & .. & .. & $\begin{array}{l}0.760 \\
(0.0000)\end{array}$ & $\begin{array}{l}-0.575 \\
(0.0000)\end{array}$ & .. & .. & .. &.. \\
\hline China & .. & .. & .. & .. & r &.. & .. & .. & .. & .. \\
\hline Chilli & $\begin{array}{l}22.894 \\
(0.0000)\end{array}$ & $\begin{array}{l}-20.667 \\
(0.0000)\end{array}$ & .. & .. & $\begin{array}{l}0.579 \\
(0.0000)\end{array}$ & $\begin{array}{l}-0.349 \\
(0.0000)\end{array}$ & .. & .. &.. & $\begin{array}{l}3.136 \\
(0.0000)\end{array}$ \\
\hline Denmark & .. & .. & .. & .. & $\begin{array}{l}0.970 \\
(0.0000)\end{array}$ & $\begin{array}{l}-0.417 \\
00.0000\end{array}$ & .. & .. & .. & .. \\
\hline France & .. & .. & -0.039 & 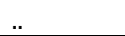 & 0.981 & -0.412 & -0.007 & .. & .. & -0.010 \\
\hline
\end{tabular}




\begin{tabular}{|c|c|c|c|c|c|c|c|c|c|c|}
\hline $\begin{array}{l}\text { Country } \\
\text { Name }\end{array}$ & LnTOTP & LnTOTP_1 & Edu & Edu_1 & LnRExp & $\begin{array}{l}\text { LnRExp } \\
\_1\end{array}$ & GEXP & GEXP_1 & $\mathbf{P}($ remi) & P(remi)_1 \\
\hline & & & 0.0041 & & $(0.0000)$ & $(0.0115)$ & $(0.0011)$ & & & $(0.0036)$ \\
\hline Germany &.. & $\begin{array}{l}1.405 \\
(0.0000)\end{array}$ &.. & .. & $\begin{array}{l}1.038 \\
(0.0000)\end{array}$ &.. & .. & .. &.. & $\begin{array}{l}0.381 \\
(0.0000)\end{array}$ \\
\hline Ghana & $\begin{array}{l}1.040 \\
(0.0000)\end{array}$ &. & .. & .. &.. & .. & $\begin{array}{l}0.002 \\
0.0490\end{array}$ & .. &.. & .. \\
\hline Hungary & .. & .. & .. & .. & $\begin{array}{l}0.830 \\
(0.0000)\end{array}$ & .. & $\begin{array}{l}-0.002 \\
(0.0064)\end{array}$ & $\begin{array}{l}-0.002 \\
(0.0067)\end{array}$ & $\begin{array}{l}-0.018 \\
(0.0000)\end{array}$ & .. \\
\hline India & $\begin{array}{l}-65.038 \\
(0.0032)\end{array}$ & $\begin{array}{l}63.691 \\
(0.0035)\end{array}$ & .. & .. &. & .. &. &. & .. &.. \\
\hline Indonesia &.. &.. & $\begin{array}{l}-0.011 \\
(0.0061)\end{array}$ & .. & $\begin{array}{l}0.848 \\
(0.0000)\end{array}$ & .. & .. & .. &.. & $\begin{array}{l}0.063 \\
(0.0000)\end{array}$ \\
\hline Iran & $\begin{array}{l}11.038 \\
(0.0034)\end{array}$ & $\begin{array}{l}-11.248 \\
(0.0025)\end{array}$ & .. & .. & $\begin{array}{l}0.573 \\
(0.0000)\end{array}$ & $\begin{array}{l}0.1400 \\
(0.0001)\end{array}$ & .. & .. & $\begin{array}{l}-0.049 \\
(0.0075)\end{array}$ &.. \\
\hline Japan & $\begin{array}{l}0.254 \\
(0.0007)\end{array}$ &. & .. & .. &. & .. & $\begin{array}{l}0.052 \\
(0.0021)\end{array}$ & $\begin{array}{l}-0.047 \\
(0.0027)\end{array}$ & .. &.. \\
\hline $\begin{array}{l}\text { Luxembour } \\
\text { g }\end{array}$ &.. & .. & .. & .. & $\begin{array}{l}0.874 \\
(0.0000)\end{array}$ & $\begin{array}{l}-0.659 \\
(0.0000)\end{array}$ & $\begin{array}{l}-0.128 \\
(0.0265)\end{array}$ & .. &.. &.. \\
\hline Malaysia & .. & .. & .. & $\begin{array}{l}-0.008 \\
(0.0049)\end{array}$ & $\begin{array}{l}0.881 \\
(0.0000)\end{array}$ & $\begin{array}{l}-0.521 \\
(0.0000)\end{array}$ & $\begin{array}{l}0.002 \\
(0.0003)\end{array}$ & $\begin{array}{l}0.001 \\
(0.0006)\end{array}$ &. & .. \\
\hline Maldives & & .. & $\begin{array}{l}-0.014 \\
(0.0437)\end{array}$ & $\begin{array}{l}0.022 \\
(0.0025)\end{array}$ & $\begin{array}{l}0.636 \\
(0.0000)\end{array}$ & $\begin{array}{l}-0.460 \\
(0.0000)\end{array}$ & .. & .. &.. & .. \\
\hline Mexico &.. & .. &. &. & $\begin{array}{l}0.753 \\
(0.0000)\end{array}$ & $\begin{array}{l}-0.609 \\
(0.0000)\end{array}$ & .. & $\begin{array}{l}-0.015 \\
(0.0007)\end{array}$ &.. & .. \\
\hline Morocco & .. & .. & $\begin{array}{l}0.059 \\
0.0178\end{array}$ & $\begin{array}{l}0.051 \\
0.0215\end{array}$ & $\begin{array}{l}0.765 \\
0.0000\end{array}$ &.. & .. &.. & $\begin{array}{l}0.027 \\
0.0007\end{array}$ & .. \\
\hline Nepal & .. & .. & .. & .. & $\begin{array}{l}0.347 \\
90.0005)\end{array}$ & $\begin{array}{l}-0.318 \\
(0.0016)\end{array}$ & .. & .. & .. & $\begin{array}{c}0.010 \\
(0.0355)\end{array}$ \\
\hline Netherland & .. & .. & .. & .. & $\begin{array}{l}0.652 \\
(0.0000)\end{array}$ & $\begin{array}{l}-0.288 \\
0.0099\end{array}$ & .. & $\begin{array}{l}0.010 \\
(0.0139)\end{array}$ & .. & .. \\
\hline Newzeland & $\begin{array}{l}5.531 \\
(0.0000)\end{array}$ & $\begin{array}{l}-5.321 \\
(0.0000)\end{array}$ & .. & .. & $\begin{array}{l}0.920 \\
(0.0000)\end{array}$ & $\begin{array}{l}-0.040 \\
0.0104\end{array}$ & .. &.. & $\begin{array}{l}-0.088 \\
(0.0215)\end{array}$ & .. \\
\hline Norway & .. & .. & .. & .. & $\begin{array}{l}0.773 \\
(0.0000)\end{array}$ & $\begin{array}{l}-0.712 \\
(0.0000)\end{array}$ & .. & & .. & $\begin{array}{l}-1.239 \\
90.0023)\end{array}$ \\
\hline Peru & $\begin{array}{l}0.685 \\
(0.0000)\end{array}$ & .. & .. & $\begin{array}{l}0.033 \\
(0.0002)\end{array}$ & $\begin{array}{l}0.508 \\
(0.0000)\end{array}$ & .. & $\begin{array}{l}0.002 \\
(0.0038)\end{array}$ & .. & .. & $\begin{array}{l}-0.019 \\
(0.0000)\end{array}$ \\
\hline Paraguay & $\begin{array}{l}-0.619 \\
(0.0000)\end{array}$ & .. & .. & .. & $\begin{array}{l}0.880 \\
(0.0000)\end{array}$ & .. & .. & .. & $\begin{array}{l}0.064 \\
(0.0008)\end{array}$ & .. \\
\hline Philippines & .. & .. & $\begin{array}{l}-0.036 \\
(0.0477)\end{array}$ & .. & $\begin{array}{l}0.807 \\
(0.0000)\end{array}$ & $\begin{array}{l}-0.776 \\
0.0000\end{array}$ & .. & .. & .. & .. \\
\hline Portugal & .. & .. & .. & .. & $\begin{array}{l}0.677 \\
(0.0000)\end{array}$ & $\begin{array}{l}-0.415 \\
(0.0006)\end{array}$ & .. & .. & $\begin{array}{l}0.001 \\
(0.0335)\end{array}$ & \\
\hline Qatar & .. & .. & $\begin{array}{l}-0.047 \\
(0.0438)\end{array}$ & $\begin{array}{l}-0.034 \\
(0.0337)\end{array}$ & $\begin{array}{l}0.425 \\
(0.0000)\end{array}$ & .. & .. & .. & $\begin{array}{l}-0.319 \\
(0.0288)\end{array}$ & $\begin{array}{l}-0.198 \\
(0.1200)\end{array}$ \\
\hline $\begin{array}{l}\text { South } \\
\text { Africa }\end{array}$ & $\begin{array}{l}-0.4982 \\
(0.0000)\end{array}$ & .. & .. & .. & $\begin{array}{l}0.944 \\
(0.0000)\end{array}$ & $\begin{array}{l}0.073 \\
(0.0582)\end{array}$ & .. & .. & .. & $\begin{array}{l}-0.424 \\
(0.0004)\end{array}$ \\
\hline Sri Lanka & $\begin{array}{l}9.954 \\
(0.0045)\end{array}$ & $\begin{array}{l}-9.919 \\
(0.0047)\end{array}$ & $\begin{array}{l}-0.044 \\
(0.0080)\end{array}$ & .. & $\begin{array}{l}0.133 \\
(0.0134)\end{array}$ & .. & .. & $\begin{array}{l}0.001 \\
(0.0059)\end{array}$ & $\begin{array}{l}-0.036 \\
(0.0057)\end{array}$ & .. \\
\hline Switzerland & $\begin{array}{l}9.480 \\
(0.0281)\end{array}$ & $\begin{array}{l}-9.514 \\
(0.0229)\end{array}$ & .. & .. & $\begin{array}{l}0.598 \\
(0.0000)\end{array}$ & $\begin{array}{l}-0.525 \\
(0.0000)\end{array}$ & .. &. &. & .. \\
\hline Sweden & $\begin{array}{l}4.812 \\
(0.0007)\end{array}$ & $\begin{array}{l}-5.029 \\
(0.0005)\end{array}$ & .. & .. & $\begin{array}{l}1.008 \\
(0.0000)\end{array}$ & $\begin{array}{l}0.069 \\
(0.0027)\end{array}$ & .. & $\begin{array}{l}0.005 \\
(0.0044)\end{array}$ & $\begin{array}{l}-0.087 \\
(0.0174)\end{array}$ & $\begin{array}{l}0.077 \\
(0.0190)\end{array}$ \\
\hline Turkey & .. & .. & .. & .. & 1.051 & -0.102 & .. & .. & .. &.. \\
\hline
\end{tabular}




\begin{tabular}{|c|c|c|c|c|c|c|c|c|c|c|}
\hline $\begin{array}{l}\text { Country } \\
\text { Name }\end{array}$ & LnTOTP & LnTOTP_1 & Edu & Edu_1 & LnRExp & $\begin{array}{l}\text { LnRExp } \\
\_1\end{array}$ & GEXP & GEXP_1 & $\mathrm{P}($ remi) & P(remi)_1 \\
\hline & & & & & $(0.0000)$ & 0.0323 & & & & \\
\hline $\begin{array}{l}\text { United } \\
\text { States }\end{array}$ & .. & .. & .. & .. & $\begin{array}{l}0.098 \\
(0.0000)\end{array}$ & $\begin{array}{l}-0.052 \\
(0.0147)\end{array}$ & .. & .. & $\begin{array}{l}-0.427 \\
(0.0300)\end{array}$ & .. \\
\hline $\begin{array}{l}\text { United } \\
\text { Kingdom }\end{array}$ & .. & $\begin{array}{l}-5.105 \\
(0.0021)\end{array}$ & .. & .. & $\begin{array}{l}0.571 \\
(0.0000)\end{array}$ & .. & $\begin{array}{l}-0.011 \\
(0.0197)\end{array}$ & .. &.. & .. \\
\hline Uruguay & .. &. & .. & .. & $\begin{array}{l}0.842 \\
(0.0000)\end{array}$ & .. & $\begin{array}{l}-0.202 \\
(0.0000)\end{array}$ & .. & .. & .. \\
\hline $\begin{array}{l}\text { Total } \\
\text { Significant }\end{array}$ & 14 & 11 & 12 & & 35 & 22 & 12 & 08 & 12 & 12 \\
\hline
\end{tabular}

The Non-Nested Encompassing model estimates the effects of GDP growth and other variables. The study includes 44 nations from 1980 to 2020. Non-nested econometric research shows that out of 44 countries, lag GDP is significant 40 times and positive in majority. GDP lag determines Gross domestic product. This means no convergence and no scale economies. The results show no indication of absolute convergence, but plenty of conditional convergence. That is, given the same policies and other circumstances, a poorer country grows faster. The results show that FDI determines economic growth in just 07 countries and is positively correlated with GDP Growth in 04 countries while negatively correlated with GDP Growth in 03 countries, implying that FDI is not a potential determinant of GDP growth in host countries. Al-Smadi (2020) links FDI and economic growth. Variable Trade Openness (TOP) is significant in 28 of 44 countries, with only one country having a positive effect in 28.Almeida et al., 2008; Barro et al., 1997.

In a few countries, labour growth is a factor of GDP. It affects 05 countries' GDP growth and is substantial for 04 countries. This variable is not regarded a possible predictor of GDP growth in 44 countries due to its small cross-country influence. Domestic income (DI) influences GDP growth in 11 countries. It has a positive impact on GDP growth in 4 countries and a negative impact in 7 . Domestic investment produces jobs and raises output levels, resulting in economic growth. Gross fix capital formation is a key predictor of GDP growth in 31 countries, and its lag value is relevant in 19. In 31 nations, GFCF is a significant factor of GDP growth. In 06 nations, its lag is linked to GDP growth [Bal et al. (2016), Uneze (2013),Total debts has no effect on economic growth in most countries. Only 11 countries out of 44 have a statistically significant lag value. Inflation is a significant predictor of GDP growth in 14 countries out of 44, while the lag value of inflation is significant in 11. The total labour force (TLF) is not a substantial factor of GDP growth in most nations. It is statistically significant in ten of the forty-four countries, while its lag value is significant in four.

Total Population (TP) is significant in 14 nations and its lag in 11. Affects Economic Growth in 4 countries. Population growth slows economic growth because most countries cannot absorb the fast increasing population, which burdens the economy and produces unemployment. Most countries do not priorities education. It is vital in 12 countries and lags in 6 . In other words, better education and productivity lead to better 
economic success. Real Exports are important for 35 countries out of 44, whereas its lag value determined economic performance for 22 countries. In most countries, exports are the key factor of growth. It doesn't affect GDP growth. It is vital in only 12 countries and lags in 8. Improving infrastructure and encouraging domestic investment supports economic growth. Personal remittances are another crucial component that can boost a country's prosperity, but only 12 of the 44 countries studied found them to be substantial. So its lag value is 12 times out of 44 . The outcomes are divided between unfavourable and positive. Affects 0 countries negatively

\section{Conclusion}

In this study six growth models have been used for analyzing the main determinants of economic growth in case of cross countries. Therefore, by using these six models we have tested them for non-nested and nested encompassing through Cox test and F-test respectively. And found that in the case of non-nested repressors in all models can explain the GDP well. And in case of nested model or full model it is concluded that model with Lag value of GDP, trade openness, population, Real Export, and gross capital formation are the main and potential determinants to boost up the Economic Growth in most of the countries. Discussing the impact of each variable separately, lag value of the variable GDP have positive and significant effect on Economic Growth in 40 countries out of the 44 country analysis. Growth of any country depend on the lag value of GDP. On the other side results of remaining variables are against the economic theories 


\section{References}

Abou-Ali, H. \& Kheir-El-Din, H. (2009). Inflation and Growth in Egypt: Is There a Threshd Effect? Middle East Development Journal, 1(1): 59-78

Anwar, S., \& Nguyen, L. P. (2010). Foreign direct investment and economic growth in Vietnam. Asia Pacific business review, 16(1-2), 183-202.

Awan, M. Z. (2010). A Nexus between foreign direct investment \& Pakistan's economy, International Research Journal of Finance and Economics, 52.

Ahmad, N., Hayat M.F., Luqman M., \& Ullah S. (2012). The Causual Links Between Foreign Direct Investment and Economic Growth in Pakistan, European Journal of Business and Economics, Volume 6, pp. 20-21

Anyanwu, J.C. (2014). Factors Affecting Economic Growth in Africa: Are There any Lessons from China, African Development Review, 26(3):468-493.

Barro, R. J. (1996). Determinants of economic growth: A cross-country empirical study. Elsevier, vol. 26(4), pages 822-824,

Bundell R., Dearden, L., Meghir, C. and Sianesi, B. (1999), "Human Capital Investment: The Returns from Education and Training to the Individual, the Firm and the Economy", Fiscal Studies, 20(1): 1-23.

Bassanini, A. \&Scarpetta, S. (2001). Does Human Capital Matter for Growth in OECD Countries? Evidence from Pooled Mean-Group Estimates", OECD Economics Department Working Papers, No. 282

Bajona, C., Kehoe, T. J., \&Ruhl, K. J. (2010). Trade liberalization, growth and productivity, University of Nottingham, UK, unpublished paper.

Biwott, P. K., Moyi, E. D., \& Khainga D. (2013).Trade Liberalization and Economic Growth: The Role of Regulatory Policies. Journal of World Economic Research, 2(3), 2013, 45-57

Cox, D. R. (1961). Tests of separate families of hypotheses.

Calero, Carla (2008). Remittances, Liquidity Constraints and Human Capital Investments In Ecuador. World Development (www.elsevier/locate/worlddev).

Gupta, S., Pattillo, C.A. \& Wagh, S. (2009). "Effect of Remittances on Poverty and Financial Development", World Development, 37(1) 
Giuliano, P., \& Ruiz-Arranz, M. (2009). Remittances, financial development, and growth. Journal of development economics, 90(1), 144-152.

Hendry, D. F. (1988). The encompassing implications of feedback versus feedforward mechanisms in econometrics. Oxford Economic Papers, 40(1), 132-149.

Hendry, D. F., \& Richard, J. F. (1983). The econometric analysis of economic time series. International Statistical Review/Revue Internationale de Statistique, 111-148. Jackson,

Hoover, K. D., \& Perez, S. J. (1999). Data mining reconsidered: encompassing and the general-to-specific approach to specification search. The Econometrics Journal, 2(2), 167-191.

Mamo, F. (2012). Economic Growth and Inflation: A panel data analysis

Silaghi, M. P., \& Medeşfălean, R. (2014). Some insights about determinants of economic growth in Romania. An empirical exercise. Theoretical \& Applied Economics, 21(6).

Uneze, E. (2013). The relation between capital formation and economic growth: evidence from sub-Saharan African countries. Journal of Economic Policy Reform, 16(3), 272-286 